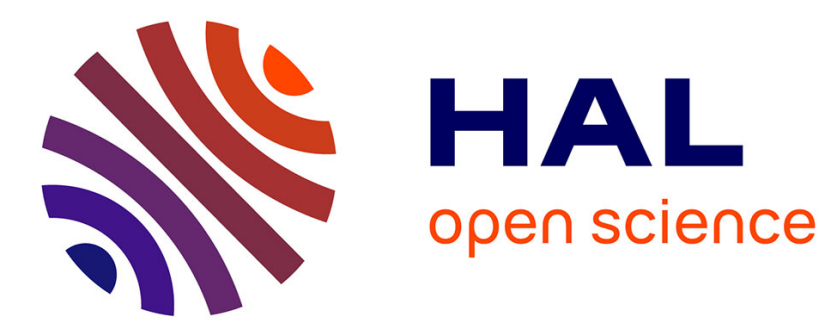

\title{
Le baptistère de Limoges (Haute-Vienne) Julien Denis
}

\section{To cite this version:}

Julien Denis. Le baptistère de Limoges (Haute-Vienne). Gallia - Archéologie de la France antique, 2006, Antiquité tardive, haut Moyen Âge et premiers temps chrétiens en Gaule méridionale (première partie) : réseau des cités, monde urbain et monde des morts, 63, pp.125-129. 10.3406/galia.2006.3291. hal-01914321

\section{HAL Id: hal-01914321 \\ https://hal.science/hal-01914321}

Submitted on 8 Jan 2020

HAL is a multi-disciplinary open access archive for the deposit and dissemination of scientific research documents, whether they are published or not. The documents may come from teaching and research institutions in France or abroad, or from public or private research centers.
L'archive ouverte pluridisciplinaire HAL, est destinée au dépôt et à la diffusion de documents scientifiques de niveau recherche, publiés ou non, émanant des établissements d'enseignement et de recherche français ou étrangers, des laboratoires publics ou privés.

\section{(a)(1) $\$$}

Distributed under a Creative Commons Attribution - NonCommercial - NoDerivatives| 4.0 


\title{
LE BAPTISTÈRE DE LIMOGES (HAUTE-VIENNE)
}

\author{
Julien DENIS
}

\begin{abstract}
Mots-clés. Baptistère paléochrétien, plan hexagonal, abside, piscine baptismale.
Résumé. La fouille archéologique, menée en 2005 à l'emplacement de l'ancien baptistère de Limoges, a permis la découverte d'un vaste édifice atypique. Ce baptistère était composé d'une salle hexagonale de plus de $10 \mathrm{~m}$ de diamètre - au centre de laquelle se trouvait une piscine baptismale de forme circulaire - et de six pièces périphériques quadrangulaires ouvrant sur l'hexagone central. L'entrée dans le baptistère s'effectuait à l'ouest. Dans le courant du haut Moyen Âge, ce baptistère fut transformé en église et agrandi d'une abside semicirculaire à son extrémité est. L'ensemble de l'édifice fut enfin méthodiquement démonté à la fin du XII ${ }^{e}$ s. ou au début du XIII ${ }^{e}$. pour laisser place à une petite église paroissiale.
\end{abstract}

Key-words. Early Christian baptistry, hexagonal plan, apse, baptismal pool.

Abstract. During the programmed excavation carried in 2005 on the spot of the former baptistry at Limoges, a vast and atypical building has been discovered. It consisted in a hexagonal room of more than $10 \mathrm{~m}$ diameter, in which a circular pool and six quadrangular rooms around it were built, opened towards the central hexagon. The entrance was situated west. During the early Middle Age, it was converted into a church and a semi-circular apse was added at the eastern end. The whole building at last has been entirely dismantled at the end of the $12^{\text {th }} \mathrm{c}$. or early $13^{\text {th }} \mathrm{c}$. and a small parish church has been established.

Translation: Isabelle FAUDUET

Schlagwörter. Frühchristliches Baptisterium, hexagonaler Grundriß, Apsis, Taufbecken.

Zusammenfassung. Eine 2005 an der Stelle des ehemaligen Baptisteriums von Limoges durchgeführte archäologische Plangrabung führte zur Aufdeckung eines ausgedehnten und atypischen Bauwerkes. Das genannte Baptisterium besteht aus einem hexagonalen Saal von mehr als 10 m Durchmesser, in dessen Mitte sich das kreisrunde Taufbecken befand, und sechs quadratischen Nebenräumen, die sich zu dem zentralen Hexagon hin öffneten. Der Zugang zum Baptisterium befand sich im Westen. Im Verlauf des frühen Mittelalters wurde das Baptisterium in eine Kirche umgewandelt und dabei am Ostende durch eine halbrunde Apsis erweitert. Gegen Ende des 12. oder zu Beginn des 13. Jahrhunderts wurde die ganze Anlage systematisch zurückgebaut, um einer kleinen Pfarrkirche Platz zu machen.

Übersetzung: Stefan WIRTH

Depuis une vingtaine d'années, l'ancienne église SaintJean-en-la-Cité, qui, jusqu'à sa destruction en 1793, se dressait devant le portail de la cathédrale de Limoges, était identifiée comme ayant succédé à un baptistère de l'Antiquité tardive (Aubrun, 1981, p. 81-84). Outre sa position au cour de la cité épiscopale et le témoignage éloquent du vocable, plusieurs autres indices laissaient peu de place au doute : non seulement cette église paroissiale (au territoire restreint, certes) n'avait disposé d'un cimetière propre qu'en 1751, mais elle avait surtout conservé le privilège de baptiser les enfants de toutes les paroisses de Limoges aux octaves de Pâques et de Pentecôte.
L'église Saint-Jean est visible sur plusieurs plans des $\mathrm{XVII}^{\mathrm{e}}$ et XVIII ${ }^{\mathrm{e}}$ s., et son emplacement, à l'extrémité est de l'actuelle place Saint-Étienne, était donc connu. En 2004, des sondages archéologiques ont permis de confirmer l'existence d'un baptistère en identifiant un édifice antérieur organisé autour d'un bassin circulaire.

La fouille archéologique programmée a été réalisée durant l'été 2005. La stratigraphie et les vestiges du baptistère (murs et sols) sont apparus extrêmement fragmentés, essentiellement en raison des occupations postérieures et de la très grande densité du réseau de canalisations urbaines. Néanmoins, les données recueillies 
permettent de retracer les caractères principaux et les évolutions de cet édifice ${ }^{66}$.

\section{L'ENVIRONNEMENT DU BAPTISTÈRE}

À partir du IV ${ }^{\mathrm{e}}$ s., l'agglomération de Limoges paraît se replier sur une hauteur située à l'est de la ville antique, le puy Saint-Étienne (Loustaud, 2000, p. 368-370). Bien que de nombreux indices, et probablement des découvertes anciennes, laissent supposer l'existence d'une enceinte, celle-ci n'a, à ce jour, toujours pas été matériellement attestée. De même, les caractères de l'occupation du nouveau site à cette époque restent largement méconnus.

C'est au centre de cette cité que s'établissent par la suite le siège épiscopal (attesté en 475) et l'ecclesia primitive (citée par Grégoire de Tours). Cette ecclesia paraît devoir être située sous l'emprise de la cathédrale actuelle (le plan de la cathédrale romane est connu et plusieurs chroniques médiévales signalent sa reconstruction à la place de l'église antérieure). Le baptistère s'inscrit également au cœur de cet espace, sur le flanc nord de la cathédrale, mais il n'y constitue pas la première occupation : le mur d'un bâtiment arasé et quelques niveaux $d u \mathrm{IV}^{\mathrm{e}} \mathrm{s}$. y ont existé antérieurement.

\section{UN ÉDIFICE DE PLAN ATYPIQUE}

Dans son premier état, le baptistère de Limoges se présentait sous la forme d'une vaste pièce hexagonale (les côtés mesurent 5,60 m de large, soit, pour la pièce, l'équivalent d'un diamètre compris entre $10 \mathrm{~m}$ et $11 \mathrm{~m}$ ) organisée autour de la piscine baptismale. Cette pièce était prolongée par six pièces quadrangulaires (des absides ?) rayonnant sur chacune des faces de l'hexagone (fig. 61).

Les pièces rayonnantes avaient toutes des dimensions intérieures de 4,60 $\mathrm{m}$ de large sur 3,60 $\mathrm{m}$ de profondeur, à l'exception des absides ouest et est qui conservaient des profondeurs respectives de $4 \mathrm{~m}$ et 4,20 m. Elles donnaient sur la pièce centrale par des ouvertures larges de $4 \mathrm{~m}$, probablement surmontées d'un arc. Le seul accès identifié du baptistère est une porte (murée par la suite) aménagée dans le mur ouest de l'abside ouest et large de 1,60 m. Enfin, de part et d'autre de cette entrée étaient également aménagées deux petites pièces trapézoïdales annexes, munies d'une porte dans leur mur occidental, mais ne communiquant pas

66. L'étude n'étant pas achevée au moment de la rédaction de cette notice, les résultats présentés ici doivent être considérés comme provisoires. avec l'intérieur du baptistère. La pièce la plus septentrionale était dallée de calcaire et abritait apparemment la canalisation d'eau alimentant la piscine baptismale. Au total, l'édifice avait une emprise au sol de $290 \mathrm{~m}^{2}$, ce qui le range parmi les plus grands baptistères des Gaules.

Les murs conservés du baptistère sont généralement peu épais (entre 0,67 $\mathrm{m}$ et 0,70 $\mathrm{m}$ de large), et faiblement fondés (0,70 m en moyenne). Ils sont appareillés en moellons très variés de gneiss ou de granite (des moellons équarris, ébauchés ou de tout-venant), probablement issus d'une récupération de matériaux. Le mortier est lui très dosé en chaux. Enfin, les chaînes d'angles sont composées de blocs de grand appareil de granite en remploi. Quant au sol de la pièce centrale, il était à l'origine composé d'un dallage calcaire reposant sur un mortier de tuileau. Un revêtement identique peut être supposé pour les absides.

Le baptistère ayant été méthodiquement démonté au début $\mathrm{du} \mathrm{XIII}^{\mathrm{e}}$ s., il ne reste plus aucun élément de décor en place. Cependant, la fouille des niveaux de démolition permet d'établir certains faits. Tout d'abord, aucune mosaïque ne paraît avoir orné l'édifice (seules huit tesselles ont été retrouvées en fouille, et aucune dans les niveaux directement associés au baptistère ou à sa démolition). En revanche, l'emploi de roches décoratives (marbres et porphyre vert) pour les revêtements muraux ne fait non seulement aucun doute, mais de plus les marbres griotte utilisés pourraient avoir fait l'objet d'une commande particulière et non pas être issus de la récupération de matériaux comme c'est le cas pour le gros œuvre. Enfin, la présence d'éléments sculptés en calcaire est assurée, malgré la récupération systématique effectuée lors de la démolition.

La fouille n'ayant livré que très peu d'éléments de datation utilisables, il faudra attendre la fin de l'étude en cours et le résultat des datations ${ }^{14} \mathrm{C}$ engagées pour préciser l'époque de construction du baptistère.

\section{LA PISCINE BAPTISMALE}

Installée au centre de l'édifice, la piscine baptismale reposait sur une épaisse semelle de béton de tuileau surmontant elle-même un puisard maçonné (fig. 62). Elle a connu au moins deux états successifs. Dans le premier état, le bassin, de forme circulaire, était large de 1,45 m avec une profondeur supérieure à $0,35 \mathrm{~m}$ (la partie supérieure de la structure n'existe plus) ; le fond, établi à la même altitude que le niveau de circulation de la pièce, était formé de dalles calcaires de belle facture, et les parois, bien qu'en grande partie masquées par le deuxième état, semblent 


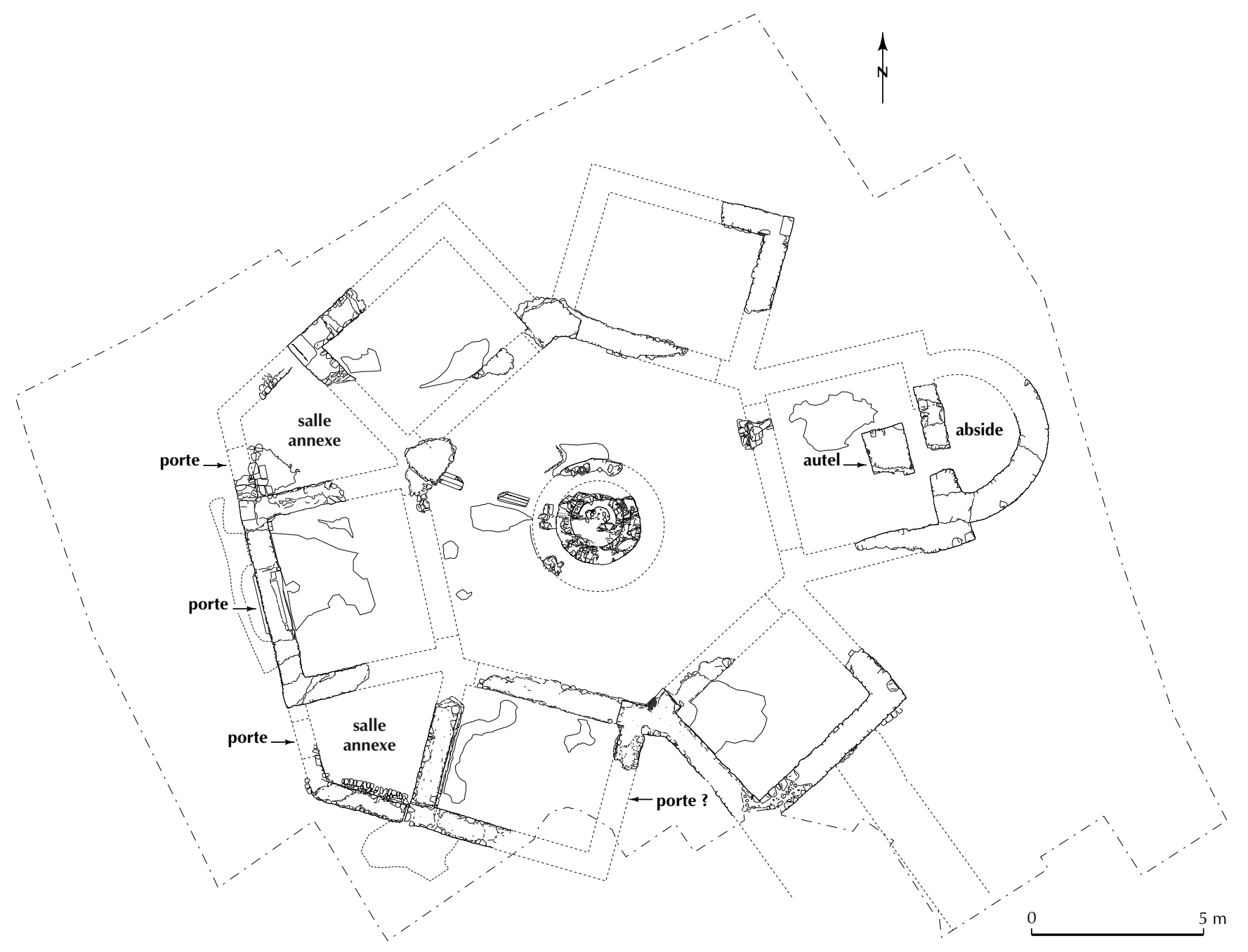

Fig. 61 - Limoges (Haute-Vienne). Le baptistère : plan cumulé des états 1 et 2 (DAO équipe Hadès).

avoir été recouvertes d'éléments calcaires posés de chant. L'alimentation en eau se faisait par une canalisation (vraisemblablement en plomb) arrivant de l'ouest et passant sous le dallage du bassin. Pour l'évacuation, une conduite verticale en plomb donnait sur le puisard.

Le deuxième état est caractérisé par un rétrécissement du bassin : une paroi de briques est bâtie à l'intérieur de la piscine, réduisant son diamètre à seulement $1,10 \mathrm{~m}$, tandis que le fond du bassin et sa paroi sont recouverts d'un enduit de mortier de tuileau rose. Dans cet état, le bassin ne dispose plus que de la conduite d'évacuation, l'alimentation ayant été supprimée (parallèlement, la conduite a été détruite et récupérée dans la petite salle annexe).

La morphologie et les dimensions successives de la piscine baptismale n'ont ici rien d'extraordinaire et s'intègrent assez bien dans les moyennes généralement constatées en Gaule, entre 1,30 m et 1,60 m. Seule la forme circulaire du bassin est un fait assez peu répandu.

\section{LA TRANSFORMATION DU BAPTISTĖRE EN ÉGLISE}

Au cours du haut Moyen Âge, l'édifice est transformé en église : à l'est, l'abside est abattue, puis reconstruite, augmentée d'une abside semi-circulaire (fig. 63). Les murs, toujours liés au mortier de chaux, sont cette fois établis en tranchée aveugle et fondés sur le substrat rocheux. Un autel maçonné est également construit, tandis que les sols des absides ouest et est sont refaits : le béton de tuileau employé ici est très chargé en brique; compact, il repose sur 


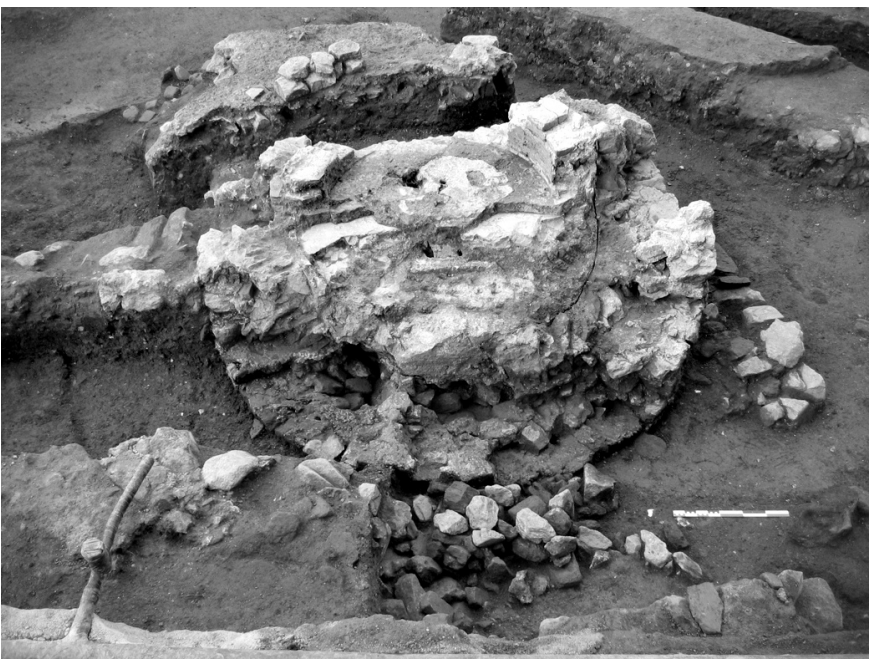

Fig. 62 - Limoges (Haute-Vienne). La piscine baptismale du baptistère (clichéJ. Denis, Hadès).

un radier massif. Par ailleurs, c'est probablement lors de la même phase de travaux que l'on procède au murage de la porte ouest. Enfin, la petite salle annexe semble avoir été détruite à la même époque.

Cependant, ces modifications majeures apportées alors au bâti, si elles n'excluent pas l'utilisation du baptistère comme église bien avant la réalisation de ces travaux, elles entérinent désormais l'abandon de son usage premier. Et, là encore, la datation par radiocarbone des charbons de bois du mortier du mur de l'abside permettra probablement de préciser les cadres chronologiques.

\section{LES DÉMOLITIONS DU DÉBUT DU XIII' $\mathrm{S}$.}

Au début du XIII ${ }^{\mathrm{e}}$ s., le baptistère est démoli. Cette démolition, très méthodique, s'est apparemment effectuée en deux temps : on a d'abord procédé à la récupération des éléments de décor, puis l'on a entamé la démolition du gros ouvre. La fouille des niveaux de démolition a permis de constater l'emploi d'échafaudages, la réalisation de tas organisés de pierres issues de la démolition, ainsi que le tri des matériaux. De nombreuses pierres paraissent ainsi avoir été remployées dans la construction de la nouvelle église Saint-Jean, et même probablement dans une maison voisine. Cette nouvelle église, bien plus petite et plus adaptée à la faible ampleur de la paroisse, fut consacrée en 1219.

$* \quad *$

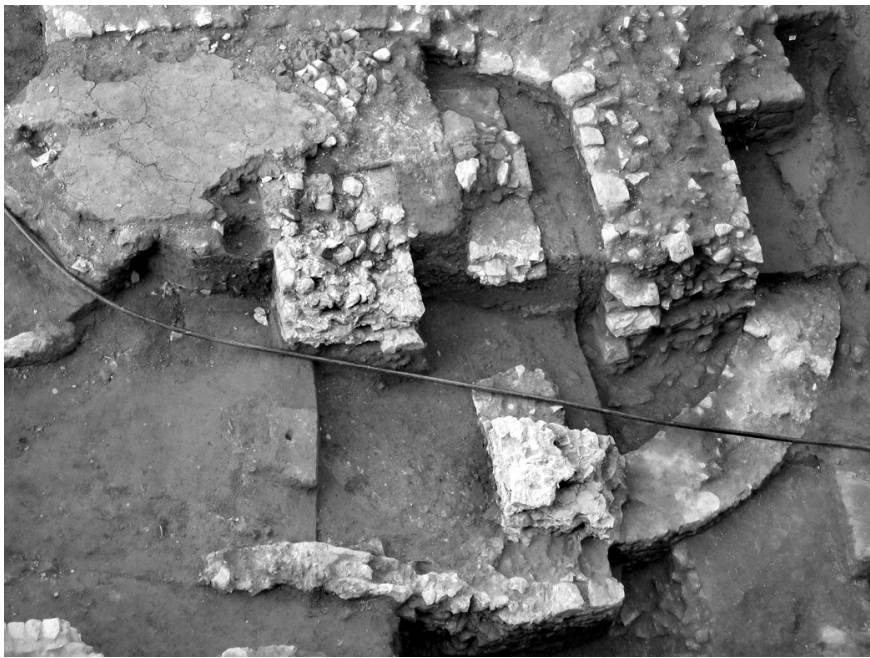

Fig. 63 - Limoges (Haute-Vienne). L'autel et l'abside semi-circulaire ajoutés à l'est du baptistère (clichéJ. Denis, Hadès).

La découverte du baptistère de Limoges vient donc compléter le corpus de ces édifices «singuliers et précieux » (Guyon, 2000, p. 67). Si le plan hexagonal utilisé ici s'avère extrêmement peu fréquent, non seulement en Gaule mais aussi dans l'ensemble de l'Empire, l'emploi d'un plan centré reste cependant la caractéristique de très nombreux baptistères. Mais le baptistère de Limoges se démarque également par un plan que l'on pourrait qualifier de rayonnant, avec l'utilisation d'absides extrêmement prononcées. Pour autant, pour ce dernier caractère, plusieurs rapprochements avec quelques édifices connus peuvent être établis en Gaule, mais également en Italie du Nord. À ce titre, le baptistère de Nevers, bien que basé sur un module octogonal, est très certainement le moins éloigné de celui de Limoges, mais les absides y alternent en plan quadrangulaire et en plan semi-circulaire. À Louin, un mausolée fouillé en 1898 et daté du $\mathrm{IV}^{\mathrm{e}} \mathrm{s}$. possède un plan hexagonal, avec des absides prononcées alternant, comme à Nevers, plan quadrangulaire et plan semi-circulaire (Duval, 1990). Sans présumer des découvertes futures (à Bourges et Clermont-Ferrand, notamment), il faudra peut-être s'interroger sur la possibilité d'un «type » de baptistère commun à la Gaule centrale, de la même manière que les baptistères provençaux présentent entre eux de fortes analogies (Guyon, 2000, p. 30).

Une autre particularité du plan est la spécificité des dimensions de l'abside ouest, par où s'effectue l'accès au baptistère, et de l'abside est (dotée d'une fonction liturgique particulière ?) par rapport aux quatre autres. La taille plus importante qui leur est accordée permet d'envisager l'hypothèse d'une 
orientation délibérée de l'édifice (là encore, le parallèle avec Nevers est assez éloquent), d'autant que, contrairement à la cathédrale, le baptistère de Limoges ne s'inscrit qu'assez mal dans la trame parcellaire antique.

Enfin, le baptistère de Limoges semble bien avoir été accolé à la cathédrale dès sa construction - et probablement même jusqu'à sa démolition - alors que l'église Saint-Jean, construite au début du XIII ${ }^{\mathrm{e}}$ s., en était séparée par une rue nouvellement créée. Outre la découverte du baptistère, cette fouille aura aussi apporté un renouvellement de la lecture du paysage urbain au XIII ${ }^{\mathrm{e}}$ s., ainsi que celui des évolutions de la cathédrale avant le chantier gothique. 